\title{
De novo Transcriptome Analysis Revealed Genes Involved in Flavonoid and Vitamin C Biosynthesis in Phyllanthus emblica (L.)
}

\author{
Avneesh Kumar, Sunil Kumar, Savita Bains, Vanya Vaidya, Baljinder Singh, Ravneet Kaur, \\ Jagdeep Kaur and Kashmir Singh *
}

Department of Biotechnology, Panjab University, Chandigarh, India

\section{OPEN ACCESS}

Edited by:

Sagadevan G. Mundree,

Queensland University of Technology,

Australia

Reviewed by:

Biswapriya Biswavas Misra

Texas Biomedical Research Institute,

USA

Andrew Wood

Southern Illinois University

Carbondale, USA

${ }^{*}$ Correspondence:

Kashmir Singh

kashmirbio@pu.ac.in

kashmir123@gmail.com

Specialty section:

This article was submitted to

Plant Biotechnology,

a section of the journal

Frontiers in Plant Science

Received: 29 March 2016 Accepted: 12 October 2016 Published: 27 October 2016

Citation:

Kumar A, Kumar S, Bains S, Vaidya V, Singh B, Kaur R, Kaur J and Singh $K$

(2016) De novo Transcriptome Analysis Revealed Genes Involved in Flavonoid and Vitamin C Biosynthesis

in Phyllanthus emblica (L.)

Front. Plant Sci. 7:1610

doi: 10.3389/fpls.2016.01610
Phyllanthus emblica is an affluent source of various therapeutic components. A few of them like vitamin $\mathrm{C}$ and flavonoids are predominant bioactive compounds that are being used in immense pharmacological applications. In-spite of numerous applications, the genomic information of this plant was limited to a few expressed sequence tags (ESTs) in DNA databases. Herein, we developed in-depth transcriptome information of $P$. emblica using Illumina Hiseq 2000 platform and characterized. A total of 31,285,965 high-quality reads were assembled into 91,288 contigs with the N50 value 358. Out of them, 47,267 contigs were functionally annotated using BLASTX search against NCBI-non-redundant (NR) protein database. Further, 31,366 contigs showed similarity with various gene ontology (GO) terms, and 1299 were related to different enzymes and biosynthetic pathways. We identified the transcripts related to each gene involved in flavonoid and vitamin C biosynthesis. Several cytochrome P450s (CYPs) and glucosyltransferases (GTs) genes involved in flavonoid biosynthesis and various other metabolic pathways were also documented. Further, 6510 transcription factors and 4420 EST derived simple sequence repeat (SSR) markers were also predicted. The present study enlightened various characteristic features of $P$. emblica genome, and provided an important resource for future molecular and functional genomics studies.

Keywords: Phyllanthus emblica, flavonoids, vitamin C, transcriptome, gene ontology, simple sequence repeats, transcription factors

\section{INTRODUCTION}

Phyllanthus emblica (syn. Emblica officinalis, family Euphorbiaceae, $n=49$ ) is a deciduous tree distributed across the subtropical and tropical regions of Asian countries such as India, China, Pakistan, Srilanka, Indonesia etc. It is a rich source of bioactive molecules like ascorbic acid (vitamin C), flavonoids, phenolics, terpenoids, tannins, rutin, curcuminoids, emblicol, phyllembelic acid, phyllembelin, emblicanin A, emblicanin B, ellagitannin, ellagic acid, gallic acid, essential amino acids, and alkaloids (Kumar et al., 2007; Poltanov et al., 2009; Krishnaveni and Mirunalini, 2010). In traditional medicines, its fruit and other parts have been extensively used in various herbal formulations to treat a variety of maladies (Perianayagam et al., 2004; Poltanov et al., 2009). Several studies suggested beneficial effects of $P$. emblica in digestion improvement, hyperthermia, blood pressure normalization, assuages asthma, hair growth, and heart and liver reinforcement. It is also 
useful in the treatment of various eye ailments, dyspepsia, gastroenteritis, anemia, hyperglycemia, fatigue, and general weakness (Perianayagam et al., 2004; Kumaran and Karunakaran, 2006; Kumar et al., 2007, 2008). The extracts of P. emblica possess antimicrobial, antioxidant, anticancer, antigenotoxic, anti-inflammatory, hepatoprotective, hypocholesterolemic, antiviral, and antifungal, hypolipidemic, antimutagenic, and immunomodulatory activities (Kumaran and Karunakaran, 2006; Kumar et al., 2007; Chatterjee et al., 2011; Singh et al., 2013). The phenolic compounds especially flavonoids in combination with vitamin $\mathrm{C}$ are the major secondary metabolites present in P. emblica.

The flavonoids are diverse class of secondary metabolites that have pivotal role in plant growth, development and defense mechanism (Dixon and Steele, 1999; Winkel-Shirley, 2001). They play critical role in the production of plant pigments and involved in various other activities including UV protection and pathogen defense, along with their nutraceutical value in the human diet (Winkel-Shirley, 2001). The structural and regulatory genes involved in flavonoid biosynthesis have been extensively characterized in various plants for their spatial and temporal regulation (Boss et al., 1996; Ban et al., 2007; Singh et al., 2008; Niu et al., 2010).

Plants are rich source of water soluble vitamin $\mathrm{C}$, which plays diverse role in various biological functions in plants and humans as well. In plants, it is involved in biosynthesis of ethylene, gibberellins, and plant pigments, cell growth regulation, acts as an enzyme cofactor in photosynthesis and various other vital functions (Smirnoff and Wheeler, 2000). Further, it is essential in ameliorating the harmful effects of reactive oxygen species derived from chloroplast in photosynthetic eukaryotes (Wheeler et al., 1998). The human beings are largely dependent upon plants for their regular uptake of vitamin $\mathrm{C}$ due to the lack of an enzyme gulonolactone oxidase involved in final step of vitamin C biosynthesis. It acts as a cofactor for enzymes involved in the post-translational hydroxylation of collagen, carnitine biosynthesis, involved in conversion of the neurotransmitter dopamine to norepinephrine, and in tyrosine metabolism (Diliberto and Daniels, 1991). It also plays vital role in regulation of iron uptake, cardiovascular functions, maintenance of cartilage, and wound healing.

The high throughput transcriptome sequencing and analyses have become a versatile method for gene discovery and expression profiling in recent years (Kalra et al., 2013; Chen et al., 2015). The Illumina sequencing technology has proven to be an exceptionally successful in a wide variety of whole-transcriptome investigations, particularly for the characterization of non-model organisms where reference genome is not available (Wilhelm et al., 2008; Tang et al., 2011; Chen et al., 2015; Kumar S. et al., 2015). Several computational tools for de novo assembly of short read sequence data and identification of genes involved in various metabolic pathways have also been demonstrated (Pertea et al., 2003; Zerbino and Birney, 2008; Grabherr et al., 2011; Fu et al., 2012).

Molecular insights into the medicinal plants have gained attention in recent years. The availability of genomic and transcriptomic data of such plants has been comprehensively reviewed by Misra (2014). Despite of high medicinal value, the genomic information of $P$. emblica is still very limited. To the best of our knowledge, only 71 ESTs were available in the National Center for Biotechnology Information (NCBI) database before the start of this work. The inadequate genomic/transcriptomic data was a major bottleneck in understanding various molecular mechanisms and biosynthetic pathways including flavonoids and vitamin $\mathrm{C}$ biosynthesis in P. emblica. Earlier, we developed a method for RNA isolation (Kumar and Singh, 2012) and cloned flavonoid 3 hydroxylase $(F 3 H)$ gene of flavonoid biosynthetic pathway using primers based PCR approach from this plant (Kumar A. et al., 2015). However, we could not get complete information about biosynthetic pathways and other molecular details using that approach. Therefore, to gain further insight into metabolic and molecular networks of this plant, the de novo transcriptome study was initiated with foremost emphasis to investigate the candidate genes involved in flavonoids and vitamin $\mathrm{C}$ biosynthesis.

\section{MATERIALS AND METHODS}

\section{Plant Material, RNA Isolation, and Transcriptome Sequencing}

Young leaves from the top aerial part of tree at the edge of branchlets (Supplementary Figure S1) and full bloom flowers were harvested from approximately 10 -year-old healthy plant of $P$. emblica growing under natural environmental conditions in the botanical garden of the Panjab University, Chandigarh, India. Samples were harvested in early morning of November month, snap frozen in liquid nitrogen, and stored at $-80^{\circ} \mathrm{C}$ till further use. Total RNA was isolated using the method described by Kumar and Singh (2012), followed by RNA purification and on column DNase I digestion using miRNA Easy kit (Qiagen, Germany). The cDNA library was prepared using TruSeq ${ }^{\mathrm{TM}}$ RNA Sample preparation kit (Illumina, USA) at Microarray core facility, Huntsman Cancer Institute, University of Utah, Salt Lake City, Utah, USA, followed by 50 cycled single end library sequencing on Illumina Hiseq 2000 sequencing platform.

\section{De novo Assembly and Sequence Clustering}

Computational analysis was carried out on HP workstation with eight cores, $2.27 \mathrm{GHz}$ Intel Xeon processor with 16 GB RAM. Data was filtered to remove adapter sequences by using the fastx_clipper tool of the FASTX Toolkit (www.hannonlab.cshl.edu/fastxtoolkit) with exact matching of target sequence. Reads passing phred quality scores $\geq 20$ (an error probability of 0.01 ) were filtered out, and unambiguous sequences ("N") were trimmed. The de novo assembly of filtered reads was performed using a short read assembler program, VELVET (Version 0.7.55) (Zerbino and Birney, 2008) followed by OASES program (Version 0.1.11) (Schulz et al., 2012) with different k-mer hash length.

After assembly, the clustering tool CD-HIT-EST was used to cluster nearly identical (>99\%) transcripts. The longest sequence within each cluster was extracted. The clustering process 
was supplemented with TGICL-CAP3 clustering program and the clustered contigs and singletons were merged to get final transcript assembly. Statistical parameters such as total transcripts, average size of transcript, transcripts having length $\geq 1000$ bp etc. were used to assess assembly quality. In order to assess the reliability of assembly, assembled sequences were further validated using previously characterized $P$. emblica gene sequences available at NCBI Genbank database. BLASTN analysis was performed for each reported sequence against the set of assembled sequences at $e$-value $10^{-5}$.

\section{Sequence Annotation and Classification}

The contigs and singletons were annotated using FastAnnotator (bioinfo.cgu.edu.tw/fastannotator_release). It utilizes Blast2GO, PRIAM, and RPS-BLAST to assign Gene ontology (GO) term, enzyme commission (EC) codes and protein domains. BLASTX was run against NCBI non-redundant (NR) protein database. The query sequences were assigned with a cut-off e-value of $10^{-5}$. Assembled transcripts were also searched against plant transcription factor database (PlnTFDB; http://plntfdb.bio.uni-potsdam.de/v3.0/downloads.php) for the identification of transcription factor families. Genes involved in flavonoid and vitamin C biosynthesis were sorted out and analyzed by BLASTX analysis.

\section{Simple Sequence Repeat (SSR) Identification}

To assess SSR markers present in the transcriptome of $P$. emblica, Microsatelite searching tool (MISA, http://pgrc.ipk-gatersleben.de/misa/) was used to mine potential microsatellites in the assembled unigenes. The mono-nucleotide (10 times), di-nucleotides (4 times), tri-, tetra-, penta-, and hexanucleotide (3 times) were searched.

\section{RESULTS AND DISCUSSION}

\section{Transcriptome Sequencing and de novo Assembly}

The high-throughput transcriptome sequencing of $P$. emblica using Illumina Hiseq 2000 (Illumina, USA) produced 32,382,864 single end reads. Since the $3^{\prime}$ end of reads are more prone to error, two bases from $3^{\prime}$ end were excluded after quality assessment. The 48 bases high quality reads were used for further analysis. After adapter trimming and removal of unambiguous sequences, 31,285,965 reads were assembled into 134,205 unique sequences and 89,242 singletons (Table 1). The de novo assembly was optimized at different k-mer lengths, in which k-mer 33 was found best with 358 bp N50 value. The longest and average contig length was 5418 and 278 bp, respectively (Table 1). Although, 100-199 bp contigs were abundant, yet 11,074 contigs had sequence length $\geq 500 \mathrm{bp}$. Contigs with $\leq 100$ bp length were discarded from further analysis. The accuracy of assembled sequences was validated by aligning them with the ESTs of $P$. emblica available at NCBI database. Significant hits were observed with ESTs with an average $82.72 \%$ similarity.
TABLE 1 | Summary of transcriptome data generated on Illumina Hiseq2000 for $P$. emblica.

Total number of single-end reads

$323,828,64$

Number of reads obtained after quality filtering

Number of assembled transcripts

Singletons

$31,285,965$

91,288

Longest contig length

89,242

Average length of transcripts (bp)

5418

278

Total base covered (bp)

$25,400,212$

\section{Sequence Clustering and Similarity Search}

The assembled sequences were further clustered by hierarchical clustering and re-assembled using Contig Assembly Program (CAP3). This step reduced the total number of uniquely assembled contigs from 134,205 to 91,288 (Supplementary Table S1 and Supplementary Figure S2). BLAST search using Fastannotator at $e \leq 1 \mathrm{e}^{-5}$ showed significant similarity of $47,267(51.7 \%)$ sequences with the NCBI-NR protein database. The e-value distribution analysis showed that $18.52 \%$ sequences matched with $\mathrm{e}<1 \mathrm{e}^{-50}$, whereas $81.48 \%$ with $e$-value ranged between $1 \mathrm{e}^{-5}$ and $1 \mathrm{e}^{-50}$ (Supplementary Figure S3). The conserved domain database search for protein functional domains indicated protein kinases, leucine rich repeats, WD40, CAZ-associated structural protein, RNA recognition motifs, mothers against decapentaplegic, pentatricho peptide repeats, zinc finger-C3HC4, and CYP450 were highly represented domains (Supplementary Figure S4). About $77 \%$ sequences showed $>60 \%$ similarity with the matched sequences in the database (Supplementary Figure S5). In terms of similarity with other species, $29 \%$ transcripts showed high similarity with the genes of Vitis vinifera, followed by Oryza sativa “japonica group” (14.35\%), Brachipodium distachyon (14.23\%), Glycine max (11.28\%), Zea mays (10.60\%), Populus trichocarpa (5.68\%), Medicago truncatula (4.84\%), and Ricinus communis (3.43\%) (Figure 1).

\section{Functional Annotation and Classification}

It was crucial to gather in-depth functional information to understand various metabolic processes. Therefore, the transcripts sequences were used for BLAST search $\left(e\right.$-value $\left.1 \mathrm{e}^{-05}\right)$ against gene ontology (GO) and enzyme classification (EC) databases (Supplementary Table S2). An overview of annotated transcripts against GO, EC, and protein domains databases is shown in Figure 2.

\section{GO Annotation}

GO analysis provides functional classification of gene, which defines the properties of genes and their products. GO has three ontologies; molecular function, cellular components, and biological processes. A total of 31,366 transcripts were annotated using GO database. In biological process category of GO (Figure 3A), oxidation-reduction process (GO: 0055114), serine family amino acid metabolic process (GO: 0009069), protein phosphorylation (GO: 0006468), regulation of transcription (GO: 0006355), and proteolysis (GO:0006508) were highly represented 


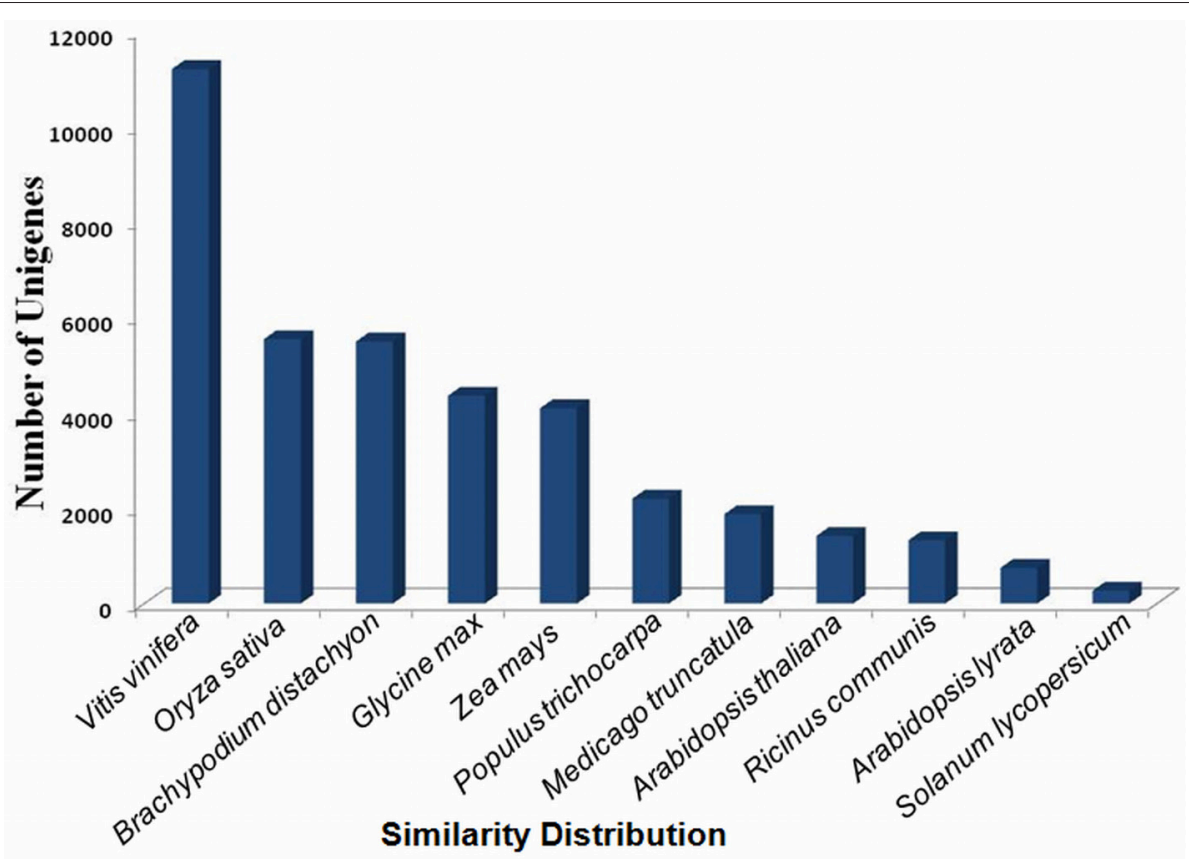

FIGURE 1 | Similarity distribution with different plant species using the NR protein database (with an $e \leq 1 e^{-5}$ ).

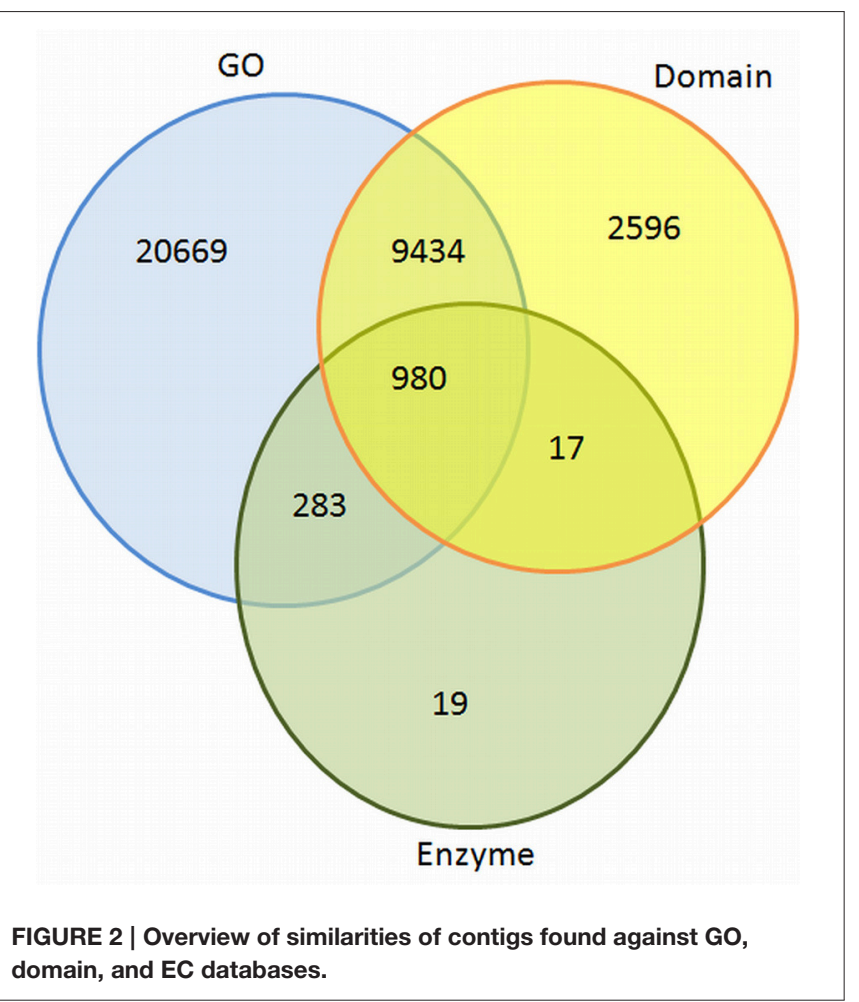

GO categories. Under cellular component, sequences related to the integral to membrane (GO:0016021), cytosol (GO:0005829), and cytoplasmic membrane-bounded vesicle (GO:0016023) were most enriched categories (Figure 3B). However, ATP binding
(GO:0005524), DNA binding (GO:0003677), protein binding (GO:0005515), and protein serine/threonine kinase activity (GO:0004674) were highly enriched in molecular function category (Figure 3C).

\section{EC Classification}

The EC annotation was obtained for 1299 transcript sequences. Top 20 abundant enzymes predicted in P. emblica transcriptome are shown in Figure 4. Non-specific serine/threonine protein kinase enzyme family (368 transcripts) members were present in high numbers. Due to the lack of tyrosine kinase receptors, tyrosine phosphorylation is less common in plants as compared to serine-threonine kinases (Mano et al., 2005). Although a number of dual-specificity kinases have been found in plants systems, but none of the true protein-tyrosine kinases (PTK) have been reported, except two PTKs being predicted in $A$. thaliana (Rudrabhatla et al., 2006; Miranda-Saavedra and Barton, 2007). Around $17 \%$ of transcripts were characterized as 2alkenal reductase (AER, EC 1.3.1.7.4). AER plays central role in the detoxification of reactive carbonyls. The reduction of $\alpha, \beta$ unsaturated bonds present in reactive carbonyls is carried out by AER and are involved in anti-oxidative defense in plants (Luan, 2002). AER plays an important role in oxidation-reduction processes, amino acid transport, and response to various stress conditions, hence the putative role of AER in P. emblica could be response to oxidative stress. About 7\% transcripts of $P$. emblica represented Ubiquitin-protein ligase (EC 6.3.2.19). They are involved in the regulation of various metabolic processes e.g., vegetative growth control mediated by hormones, plant reproduction, stress tolerance, and DNA repair (Mazzucotelli et al., 2006). E3 ubiquitin-ligases are also known to regulate 
A

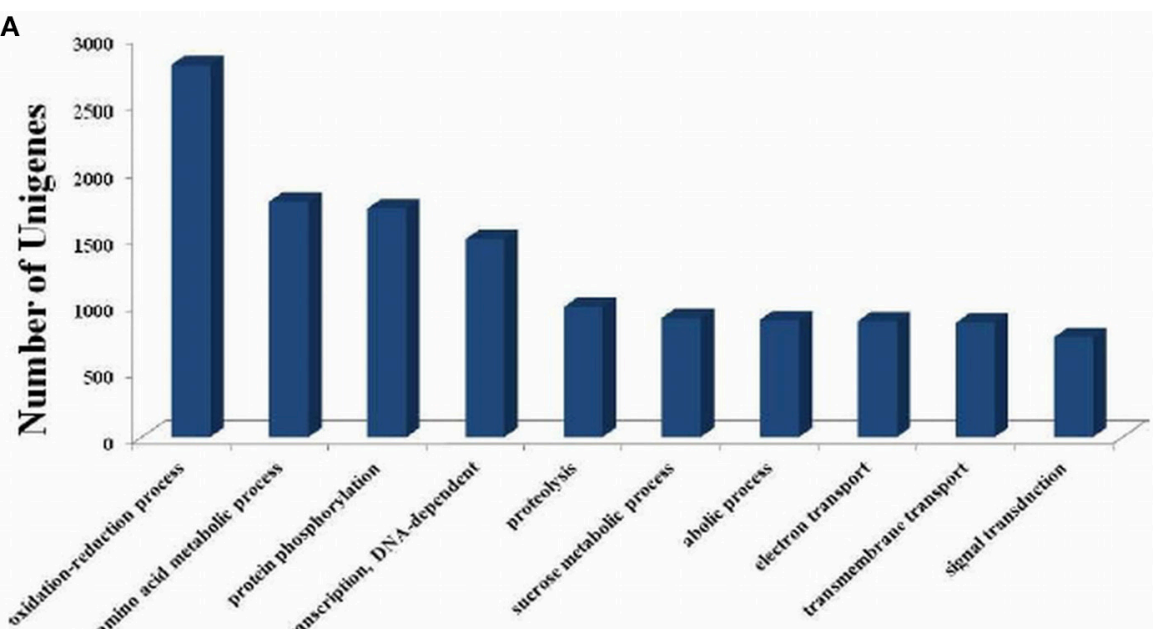

GO: Biological Process

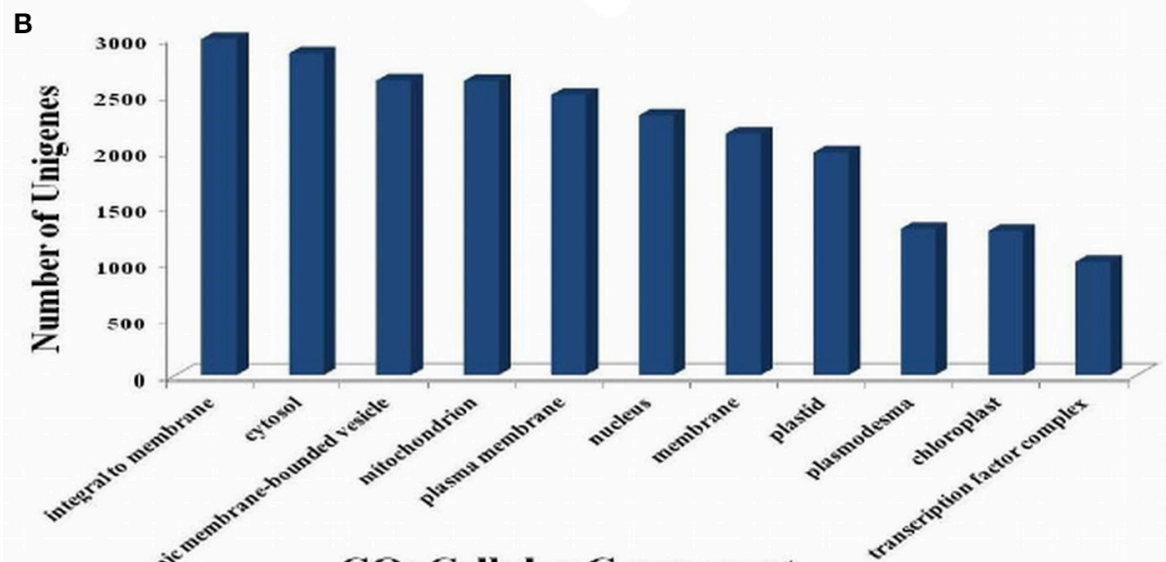

GO: Cellular Component

C

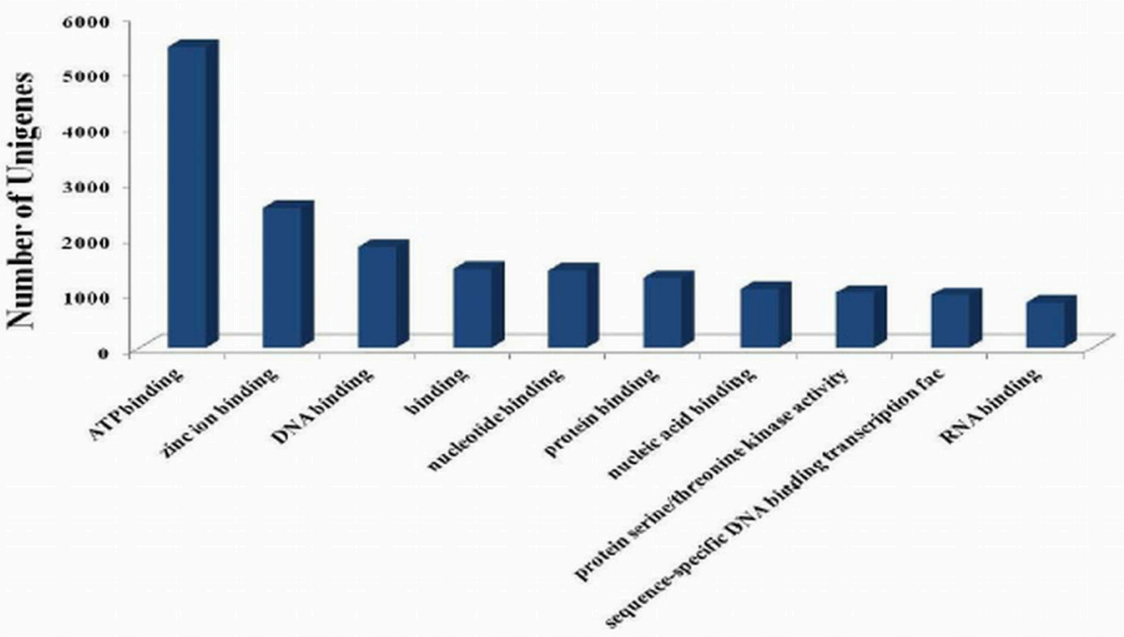

GO: Molecular Function

FIGURE 3 | Gene Ontology (GO) classification of the $\boldsymbol{P}$. emblica transcriptome. GO term are summarized into three main categories (A-biological process, B-cellular component, and $\mathbf{C}$-molecular function) based on significant hits of unigenes against the NR database. 
signaling pathways initiated by $\mathrm{ABA}$ induced stress (Lyzenga et al., 2011).

\section{Analysis of the Transcripts Encoding Transcription Factors (TFs)}

Transcripts encoding TFs were identified by sequence comparison to known TF gene families. In total, 6510 (7.10\%) putative transcripts showing similarity to TF genes were identified in P. emblica (Supplementary Table S3). These included TF families like C3H, PHD, FAR1, MADS, SET, SNF2, MYB, bHLH etc (Figure 5). Out of them, C3H (601 transcripts), PHD (447 transcripts), and FAR1 (375 transcripts) were most abundant, and GNAT and ABI3VP1 were least represented. Several $\mathrm{C} 3 \mathrm{H}$ proteins have been reported to participate in developmental responses and hormonal pathways. Thus, $\mathrm{C} 3 \mathrm{H}$ is expected to play significant role in stress responses and various metabolic processes in P. emblica, also.

The flavonoid pathway genes are mainly regulated at transcription level (Winkel-Shirley, 2001). Several TFs regulating flavonoid biosynthesis have been identified in plants (Davies and Schwinn, 2003; Allan et al., 2008; Palapol et al., 2009; Niu et al., 2010). The R2R3-MYB and bHLH TFs form the MBW complex with the WD40 proteins, which may regulate the transcription of various genes of flavonoid biosynthetic pathway in plants. This regulation is via specific binding to motifs in the promoter region (Hernandez et al., 2004; Hartmann et al., 2005; Dare et al., 2008). The basic helix loop-helix (bHLH) family of TFs is one of the most abundant and highly conserved in plant kingdoms. These bind the E-box (CANNTG), although most plant bHLHs specifically recognize the so-called G-box (CACGTTG). The bHLH proteins are known to regulate biological processes i.e., light signaling, hormone signaling, wound and drought stress response, organ, and tissue development. The R2R3-MYB and bHLH TFs responsible for the anthocyanin accumulation have been well characterized (Ban et al., 2007; Espley et al., 2007). In grapes R2R3-MYB TF, VvMYBA activates the UDP-glucose:flavonoid3-O-glycosyltranferase (UFGT) gene, which plays a key role in color development (white and red) in grape skin (Boss et al., 1996; Kobayashi et al., 2002). PpMYB10 of peach bind to dihydroflavonol 4-reductase (DFR) promoter and activated the anthocyanin biosynthesis in tobacco and Arabidopsis (Lin-Wang et al., 2010).

In Arabidopsis, three closely related MYBs, AtMYB11, AtMYB12, and AtMYB111 regulate AtFLS1 and other steps for production of flavonol glucosides (Mehrtens et al., 2005; Stracke et al., 2007). These genes share significant similarity and form subgroup 7 of $R 2 R 3-M Y B$ gene family. Due to the functional similarity amongst MYB11, MYB12, and MYB111, they show target specificity for flavonoid biosynthetic pathway genes such as CHS, CHI, F3H, and FLSI. The FLS is regulated by light and UV exposure via activation of MYB TFs in grapes and maize, respectively (Czemmel et al., 2009; Ferreyra et al., 2012). At least four MYB TFs ( $V v M Y B 5 a, V v M Y B 5 b$, $V v M Y B P A 1$, and $V v M Y B P A 2)$ are reported in grapes that regulate key steps of the flavonoid pathway. They affect accumulation of proanthocyanidins in leaves, flowers and in early berry development, before the véraison stage (Terrier et al., 2009).

\section{Analysis of Metabolic Pathway Genes Flavonoid Biosynthesis Pathway}

All the genes associated with flavonoid biosynthesis pathway were detected in the transcriptome data of $P$. emblica (Figure 6) with multiple transcripts for each gene (Supplementary Table S4). Flavonoids are synthesized via the phenylpropanoid pathway, wherein phenylalanine gets deaminated to form cinnamic acid by phenylalanine ammonia lyase (EC 4.3.1.24, 19 transcripts). The hydroxylation of cinnamic acid by cinnamate 4-hydroxylase (EC 1.14.13.11, 2 transcripts) produces $p$-coumaric acid, which subsequently converted to $p$-coumaroyl-CoA by 4-coumaroyl CoA ligase (EC 6.2.1.12, 5 unigenes). Further, Chalcone synthase (EC 2.3.1.74, 24 unigenes) catalyses chalcone production by condensation of 4-coumaroyl-Co A and malonyl-Co A in 1:3 ratio. After two-steps of condensation catalyzed by chalcone synthase and chalcone isomerase (EC 5.5.1.6, 12 unigenes), a flavanone called naringenin is produced. It acts as a precursor of many flavonoid and isoflavonoid compounds. The naringenin is oxidized by flavanone 3 hydroxylase (EC 1.14.11.9, 12 unigenes) into dihydroflavonols. The hydroxylation of naringenin by flavonoid $3^{\prime}$-hydroxylase (EC 1.14.13.21, 9 unigenes) and flavonoid $3^{\prime}, 5^{\prime}$-hydroxylase (EC 1.14.13.88, 0 unigene) yields eriodictyol and dihydrotricetin, respectively. Naringenin, eriodictyol, and dihydrotricetin are flavanones which are involved in plant stress responses. Flavone synthase (EC 1.14.11.22, 1 unigene) catalyzes the conversion of flavanones to flavones, and flavanone 3-hydroxylase (EC 1.14.11.9, 10 unigenes) can convert these flavanones to dihydroflavonols. The dihydroflavonols lead to the production of flavonols and flavan- 3, 4-diols (leucoanthocyanidin), by the actions of flavonol synthase (EC 1.14.11.23, 12 unigenes) and dihydroflavonol 4-reductase, a NADPH-dependent enzyme (EC 1.1.1.219, 23 unigenes), respectively. Leucoanthocyanidins are converted either to anthocyanidins through the action of anthocyanidin synthase (EC 1.14.11.19, 5 unigene) or reduced to catechins by anthocyanidin reductase (EC 1.3.1.77, 2 unigenes). Catechins are bioprospecting flavonoids as they have huge manifestation in human health and are also involved in plant growth and survival. Hence, they might be potential targets for metabolic engineering (Rani et al., 2012).

These annotations were useful in predicting the molecular functions of unigenes and constructing the metabolic pathways in P. emblica. The knowledge of flavonoid biosynthetic pathways along with the regulatory TFs (such as MYB, $b H L H$, and WD40type) rendered metabolic engineering much simplified for the production of various essential metabolites.

Isoflavonoids are an important subclass of flavonoids being involved in plant defense and nodulation (Dixon and Steele, 1999). Certain transcripts associated with isoflavonoid metabolism such as isoflavone 7-O-methyltransferase, 2-hydroxy isoflavanone synthase, isoflavone $2^{\prime}$-hydroxylase, isoflavone 4'-Omethyltransferase, and isoflavone reductase were also present in our data. 


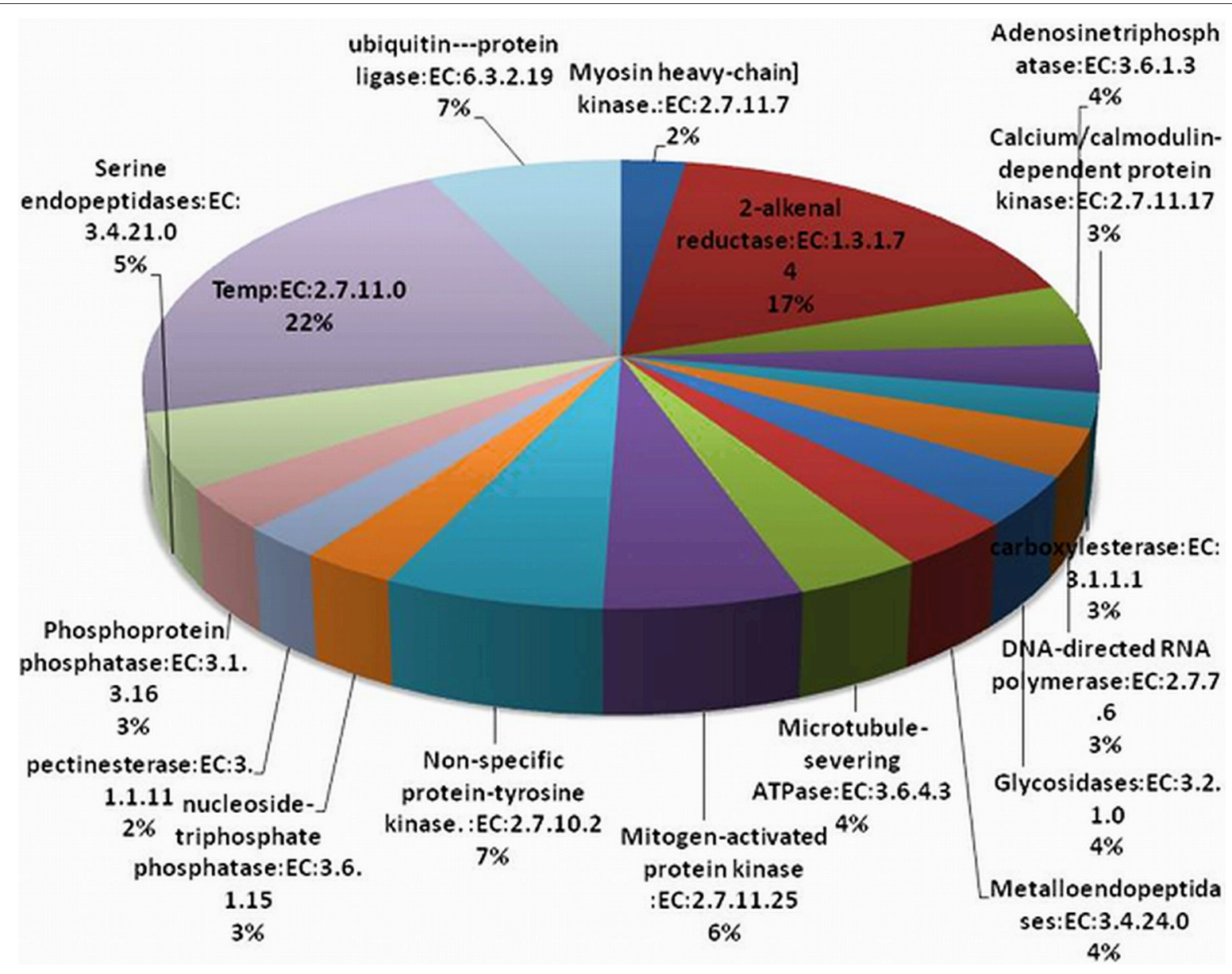

FIGURE 4 | Abundance of enzyme classes (Top 20) in P. emblica transcriptome. Area under each pie represents the \% value of actual number of transcripts.

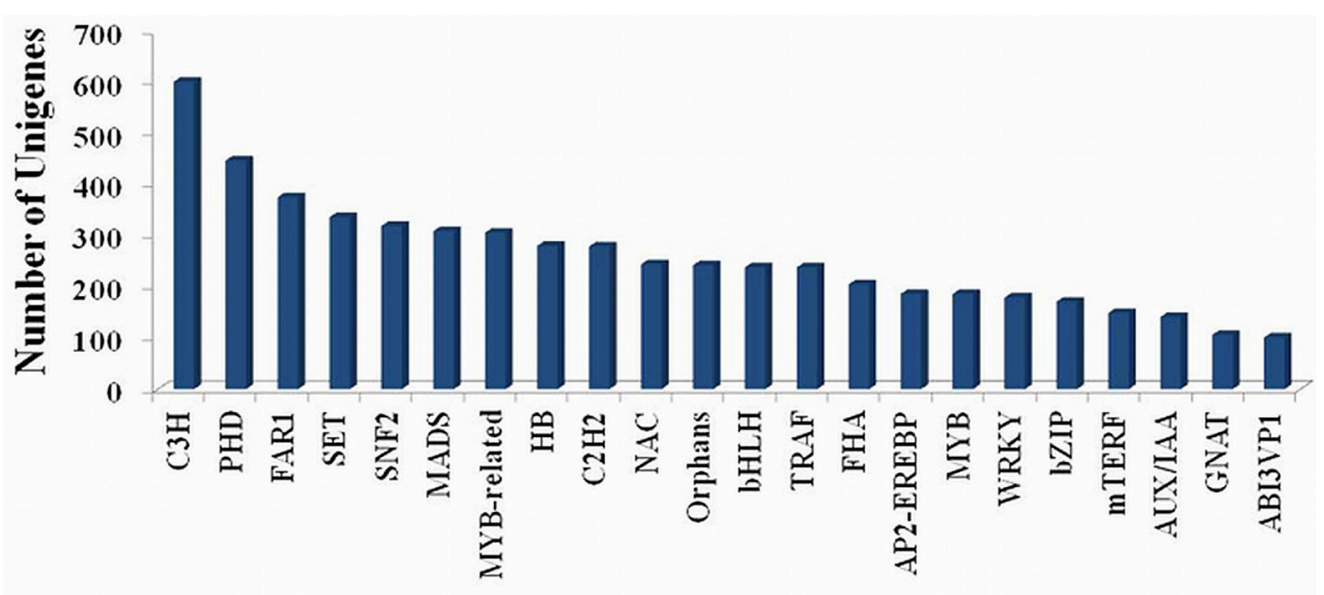

\section{Transcription Factor Family}

FIGURE 5 | Number of unigenes of $\boldsymbol{P}$. emblica matching with different transcription factor families. Top 22 families are shown here and rest are mentioned in Supplementary Table S3. 


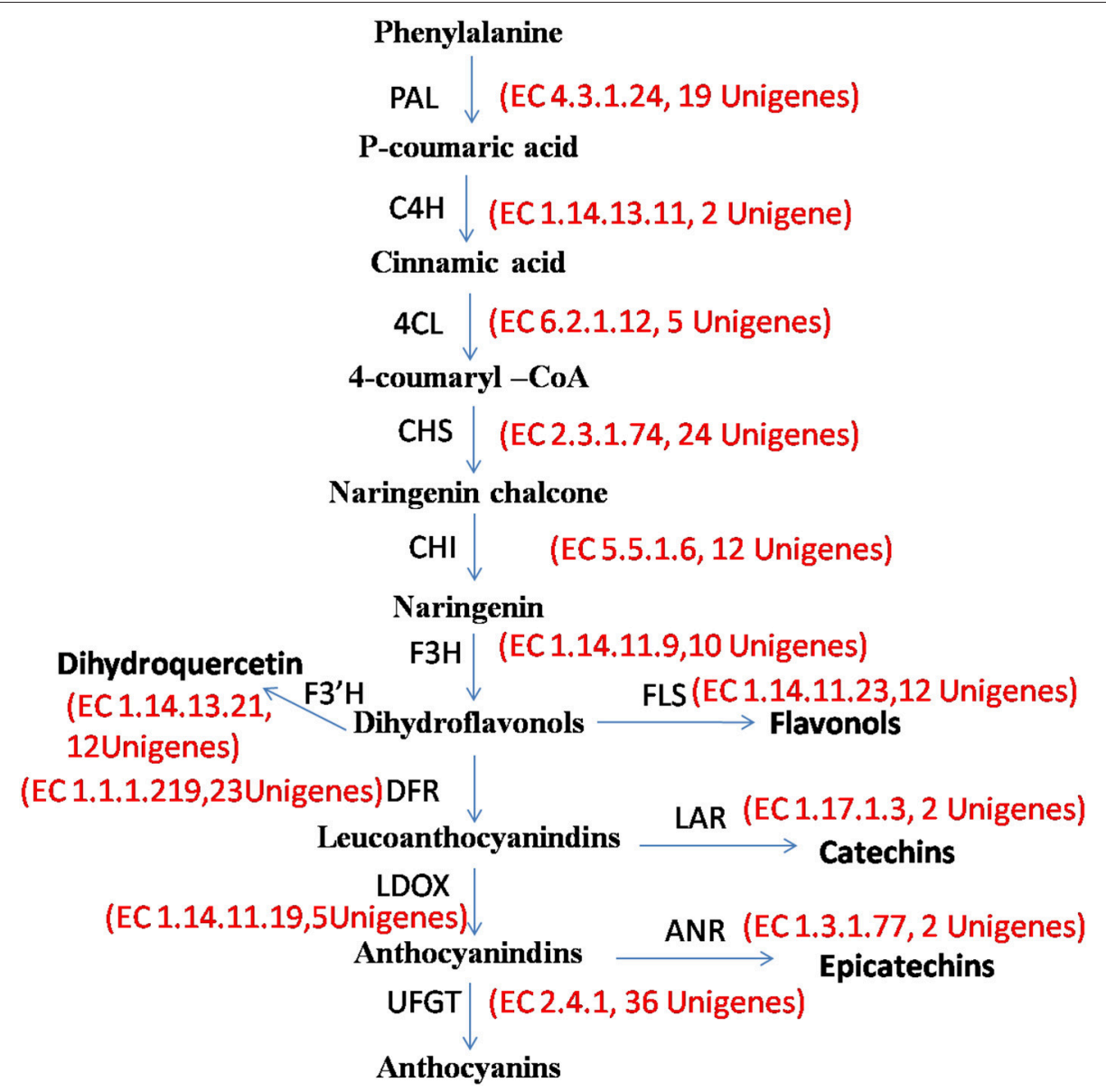

FIGURE 6 | $\boldsymbol{P}$. emblica unigenes involved in flavonoid biosynthesis pathway. Number in brackets following EC number indicates the number of unigenes identified for the corresponding gene.

\section{Discovery of Transcripts Encoding for CYPs and GTs}

CYP450s form the largest superfamily of enzymes because of its prevalence in plant systems and implications in plant metabolism. The role of CYP450s in catalyzing a wide range of regio-specific, stereospecific and irreversible steps involved in plant metabolite biosynthesis is well documented (Renault et al., 2014). A total of 214 unique transcripts were annotated as CYP450s (Supplementary Table S5). Amongst all, cinammate 4hydroxylase (2 unigenes), flavanone 3-hydroxylase (EC 1.14.11.9, 12 unigenes), flavone synthase II (EC 1.14.11.22, 3 unigene), and flavonoid 3'-hydroxylase (EC 1.14.13.21, 9 unigenes) are known to be involved in flavonoid biosynthesis.

The P450 monooxygenases are heme protein-dependent mixed-function oxidase systems. They utilize NADPH/NADH to reduce atmospheric dioxygen and yield an organic substrate along with a water molecule. They are involved in several processes such as hydroxylation, dealkylation, dimerizations, epoxidation, isomerization, deamination etc. Schuler and WerckReichhart (2003).

Activities of P450s are categorized into two classes- first exists in biosynthetic pathways and second in detoxification pathways (Schuler, 1996; Chapple, 1998). They play important role during synthesis of lignin, flavonoids, coumarins, sinapoyl esters, isoflavonoids, hydroxamic acids, glucosinolates, terpenes, gibberellins, brassinosteroids, auxin, and oxygenated fatty acids. In addition to these known biosynthetic activities, plant P450s are also responsible for catabolizing a range of endogenous and toxic exogenous compounds encountered in the environment such as herbicides, insecticides, and some pollutants (Werck-Reichhart et al., 2000; Harvey et al., 2002).

Several unigenes encoding different types of glycosyltransferases were also found in our dataset (Supplementary Table S6). Out of them, UFGTs supposed to be involved in flavonoid biosynthesis included flavonoid glucosyltransferase, UDP-glucose:isoflavone 7-O-glucosyltransferase, tetrahydroxychalcone glucosyltransferase, and anthocyanidin 3-O-glucosyltransferase.

The glycosylation of anthocyanidin resulted into increase in stability, decrease in reactivity, and change in its spectral features; otherwise anthocyanidins are highly reactive and inherently unstable. Major increase in anthocyanin accumulation is attributed to higher mRNA levels of UFGT, CHS, and F3H genes (Prior and Wu, 2006; Petrussa et al., 2013). UFGT is 


\section{D-GlucoseD-Fructose-6-phosphate \\ Hexokinase $\downarrow$ (23 Unigenes) \\ D-Glucose-6-phosphate \\ Phosphomannose \\ isomerase \\ (5Unigenes) \\ D-Mannose-6-phosphate \\ Phosphomannomutase $\downarrow$ (2 Unigenes)}

\section{D-Mannose-1-phosphate}

GDP-D-Mannose Pyrophosphorylase

(25 Unigenes)

\section{GDP-D-Mannose}

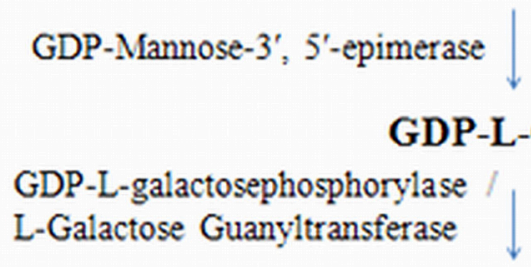

(12 Unigenes)

L-Galactose-1-Phosphate

L-Galactose-1-P Phosphatase $\downarrow$ (7 Unigenes)

\section{L-Galactose}

\section{L-Galactose Dehydrogenase $\downarrow$ (2 Unigenes)}

\section{L-Galactono-1,4-lactone}

\begin{tabular}{l|l} 
L-Galactono-1, 4- & (2 Unigenes) \\
lactone Dehydrogenase &
\end{tabular}

\section{L-Ascorbic acid}

FIGURE $7 \mid$ P. emblica unigenes involved in LAA (vitamin C) biosynthetic pathway. Number in brackets indicates the number of unigenes identified for the corresponding gene.

the final gene in anthocyanin pathway and play vital role in anthocyanin biosynthesis and accumulation. The UFGT mediated transfer of the glucosyl moiety from UDP-glucose to hydroxyl groups of anthocyanidins is shown crucial for their stability and solubility. The expression of UFGT is reported to be controlled by various transcription factors like MYB10, MYB123, and bHLH3 (Ravaglia et al., 2013).

\section{Vitamin C (L-Ascorbic Acid) Biosynthesis}

$P$. emblica contains high levels of vitamin $\mathrm{C}$, therefore transcriptome analysis is an indispensable tool to investigate the genes involved in its biosynthesis. Transcripts related to each gene involved in biosynthesis of L-Ascorbic Acid (AsA) were identified in the current study (Figure 7). The number of contigs matching with the pathway genes varies from 2 for $L$ galactose dehydrogenase to more than 20 for Hexokinase and
GDP-D-Mannose pyrophosphorylase (Supplementary Table S7). AsA biosynthesis in plants occurs through various routes but activation of each pathway is dependent upon species and developmental stages of the plant. The first proposed pathway for AsA biosynthesis proceeds through GDP-D-mannose and Lgalactose (Wheeler et al., 1998). In 1963, it was demonstrated in strawberry fruits, where 6-carbon labeled molecule D-glucose converted into AsA without cleavage of the carbon chain. Later on, it was postulated that L-galactono-1, 4-lactone is the immediate precursor for AsA.

D-Glucose is the main precursor for ascorbic acid biosynthesis, which gets converted into $\mathrm{D}$-glucose 6-phosphate by the enzyme hexokinase (EC 2.7.1.1), a component of glycolysis. The glucose ring is phosphorylated by addition of a phosphate group derived from ATP. D-Glucose-6-phosphate isomerized into D-fructose-6-Phosphate with the action of 
phosphoglucose isomerase (EC 5.3.1.9, 364 unigenes) by rearrangements of carbon oxygen bond to transform 6-C ring into 5-C ring. It further converted mannose-6-phosphate by phosphomannose isomerase (EC 5.3.1.8, 5 unigenes). Subsequently phosphomannomutase (EC 5.4.2.8, 3 unigenes) transfers the phosphate group from 6-carbon position to 1carbon position to produce D-mannose-1-phosphate. Then, GDP-D-mannose pyrophosphorylase (EC 2.7.7.22, 19 unigenes) adds one glycosyl unit to produce GDP-D-mannose, which epimerizes at $3^{\prime}$ and $5^{\prime}$ positions to yield GDP-L-galactose by enzyme GDP-mannose-3', 5'-epimerase (EC 5.1.3.18, 23 unigenes). GDP-mannose pyrophosphorylase is reported to have significant role in regulation of AsA biosynthesis. In fact, a correlation between mRNA levels of GDP-mannose pyrophosphorylase and AsA levels has been documented in many plant species (Badejo et al., 2007, 2009).

The conversion of GDP-L-galactose into L-galactose-1phosphate is catalyzed by GDP-L-galactose phosphorylase (EC 2.7.7.69, 14 unigenes). L-galactose-1-phosphate is regarded as the first metabolite dedicated to AsA biosynthesis. Hence, this step represents the first committed step in the whole pathway. Further, L-galactose-1-phosphate leads to the formation of Lgalactose by the activity of L-galactose-1-P phosphatase (EC 3.1.3.25, 14 unigenes). L-galactono-1, 4-lactone is produced by the activity of L-galactose dehydrogenase (EC 1.1.1.316, 14 unigenes) from L-galactose. It is postulated that regulation of L-galactose dehydrogenase expression is light dependent and light-dependent changes in respiration might directly affect their activity (Tamaoki et al., 2003; Bartoli et al., 2006). Finally, Lascorbic acid is produced from L-galactose by the action of Lgalactono-1,4-lactone dehydrogenase (EC 1.3.2.3, 5 unigenes) enzyme, which is highly specific for L-galactono-1, 4-lactone.

The enzyme GDP-mannose $3^{\prime}, 5^{\prime}$-epimerase is responsible for the catalytic conversion of GDP-D-mannose into GDP-Lgalactose. A novel compound, GDP-L-gulose is also produced by the $5^{\prime}$-epimerization of GDP-D-mannose. Therefore, it was postulated that the GDP-mannose-3', 5'-epimerase enzyme catalyses distinct epimerization reactions depending upon the molecular form of enzyme leading to the formation of either GDP-L-galactose or GDP-L-gulose (Wolucka and Van Montagu, 2003). Here, we found a new branch point of this pathway in plants and a connecting link with the pathway operating in animals. Cats and dogs can synthesize their own vitamin $C$ unlike humans, because human cells cannot perform the conversion of 1-gulono-1,4-lactone into ascorbic acid, which is catalyzed by the enzyme gulonolactone oxidase. It was observed that the gene gulonolactone oxidase is present in humans, but it is a non-functional pseudogene because of accumulation of several mutations over the time (De Tullio, 2010).

\section{SSR Analysis}

A total of 4420 SSRs were identified in 4079 transcripts of $P$. emblica, in which 314 sequences contained more than 1 SSR (Table 2). With a frequency of over 43.5\% (1925/4420), dinucleotides were most abundant SSRs, which was followed by tri-nucleotides $(33.2 \%, 1469 / 4420)$, tetra-nucleotides $(0.045 \%$, $20 / 4420)$ and penta-nucleotides $(0.013 \%, 6 / 4420)$. The results
TABLE 2 | Simple sequence repeats (SSRs) identified in transcripts of $P$. emblica.

\begin{tabular}{lc}
\hline SSR mining & Number \\
\hline Total number of sequences examined: & 91,288 \\
Total size of examined sequences (Mb): & 25.42 \\
Total number of identified SSRs: & 4420 \\
Number of SSR containing sequences: & 4079 \\
Number of sequences containing more than one SSR: & 314 \\
Number of SSRs present in compound formation: & 248 \\
DISTRIBUTION OF SSRs IN DIFFERENT REPEAT TYPES & \\
Unit size & Number of SSRs (\%) \\
Mononucleotide & 986 \\
Dinucleotide & 1925 \\
Trinucleotide & 1479 \\
Tetranucleotide & 20 \\
Pentanucleotide & 6 \\
Hexanucleotide & 14 \\
\hline
\end{tabular}

TABLE 3 | Simple sequence repeats (SSRs) identified in the genes involved in flavonoid and vitamin $\mathrm{C}$ biosynthesis.

\begin{tabular}{lll}
\hline $\begin{array}{l}\text { Gene name } \\
\text { FLAVONOID BIOSYNTHESIS }\end{array}$ & Contig ID & SSR motif \\
$\begin{array}{l}\text { Phenylalanine ammonia lyase } \\
\text { Chalcone isomerase }\end{array}$ & NODE_135356 & $(\mathrm{A})_{11}$ \\
$\begin{array}{l}\text { Dihydroflavonol reductase } \\
\text { VITAMIN C BIOSYNTHESIS }\end{array}$ & NODE_45303 & $(\mathrm{GA})_{20}$ \\
$\begin{array}{l}\text { Phosphoglucoseisomerase } \\
\text { GDP-D-Mannose pyrophosphorylase }\end{array}$ & NODE_119675 & $(\mathrm{CT})_{22}$ \\
$\begin{array}{l}\text { L-Galactose-1-P phosphatase } \\
\text { L-Galactose dehydrogenase }\end{array}$ & NODE_25565 & $(\mathrm{GCAT})_{3}$ \\
\hline
\end{tabular}

indicated that transcripts containing SSR markers were indeed abundant in P. emblica. In particular, dinucleotide SSRs were identified within the sequences of flavonoid biosynthetic pathway genes such as $P A L, C H I$, and DFR. In vitamin C biosynthesis pathway, SSR motifs were identified in four genes (Table 3). Additionally, di-nucleotide to tetra-nucleotide repeats were predicted in various other genes. Therefore, in future, research on various isoforms and activities of genes involved in flavonoid and vitamin $\mathrm{C}$ biosynthesis may be helpful in explaining, why $P$. emblica has such a high amount of flavonoids and vitamin $\mathrm{C}$ ?

\section{DATA ARCHIVING STATEMENT}

Transcriptome data of this study can be accessed at NCBI SRA database under SRA ID SRP075209 (Bioproject PRJNA313483).

\section{AUTHOR CONTRIBUTIONS}

Conceived and designed the experiments: KS Performed the experiments: AK, SK, SB Analyzed the data: SK, SB, VV, RK 
Contributed reagents/materials/analysis tools: KS, JK, RK, SK Wrote the paper: KS, RK, BS.

\section{ACKNOWLEDGMENTS}

We are thankful to Dr. Brian Dalley, Director, Microarray core facility, Huntsman Cancer Institute, University of Utah, Salt Lake City, USA for mRNA library preparation and high-throughput sequencing. AK and SK are thankful to CSIR, India for awarding junior and senior research fellowship. Authors are thankful to Dr. Shikha Kalra and Dr. Dharam Singh for critically reading the manuscript and giving their valuable suggestions.

\section{SUPPLEMENTARY MATERIAL}

The Supplementary Material for this article can be found online at: http://journal.frontiersin.org/article/10.3389/fpls.2016. 01610/full\#supplementary-material

Supplementary Figure $S 1 \mid$ Herbarium record of the $P$. emblica plant deposited in the herbarium of Botany department of Panjab University, Chandigarh, India (recford no 21074). Position of young leaves have been marked.

\section{REFERENCES}

Allan, A. C., Hellens, R. P., and Laing, W. A. (2008). MYB transcription factors that colour our fruit. Trends Plant Sci. 13, 99-102. doi: 10.1016/j.tplants.2007.11.012

Badejo, A. A., Tanaka, N., and Esaka, M. (2007). Cloning and expression of GDP-D-mannose pyrophosphorylase gene and ascorbic acid content of acerola (Malpighia glabra L.) fruit at ripening stages. Plant Physiol. Biochem. 45, 665-672. doi: 10.1016/j.plaphy.2007.07.003

Badejo, A. A., Tanaka, N., and Esaka, M. (2009). Analysis of GDP-D-mannose pyrophosphorylase gene promoter from acerola (Malpighia glabra) and increase in ascorbate content of transgenic tobacco expressing the acerola gene. Plant Cell Physiol. 49, 126-132. doi: 10.1093/pcp/pcm164

Ban, Y., Honda, C., Hatsuyama, Y., Igarashi, M., Bessho, H., and Moriguchi, T. (2007). Isolation and functional analysis of a MYB transcription factor gene that is a key regulator for the development of red coloration in apple skin. Plant Cell Physiol. 48, 958-970. doi: 10.1093/pcp/pcm066

Bartoli, C. G., Yu, J., Gómez, F., Fernandez, L., McIntosh, L., and Foyer, C. H. (2006). Inter-relationships between light and respiration in the control of ascorbic acid synthesis and accumulation in Arabidopsis thaliana leaves. J. Exp. Bot. 57, 1621-1631. doi: 10.1093/jxb/erl005

Boss, P. K., Davies, C., and Robinson, S. P. (1996). Analysis of expression of anthocyanin pathway genes in developing Vitis vinifera L. cv shiraz grape berries and the implications for pathway regulation. Plant Physiol. 111, 1059-1066. doi: 10.1104/pp.111.4.1059

Chapple, C. (1998). Molecular-genetic analysis of plant cytochrome P450dependent monooxygenases. Ann. Rev. Plant Physiol. Plant Mol. Biol. 49, 311-343. doi: 10.1146/annurev.arplant.49.1.311

Chatterjee, A., Chattopadhyay, S., and Bandyopadhyay, S. K. (2011). Biphasic effect of Phyllanthus emblica L extract on NSAID-induced ulcer: an antioxidative trail weaved with immunomodulatory effect. Evid. Based Complement. Alternat. Med. 2011:146808. doi: 10.1155/2011/146808

Chen, J., Wu, X.T., Xu, Y. Q., Zhong, Y., Li, Y., Chen, J., et al. (2015). Global transcriptome analysis profiles metabolic pathways in traditional herb Astragalus membranaceus Bge. var. mongolicus (Bge.) Hsiao. BMC Genomics 16:S15. doi: 10.1186/1471-2164-16-S7-S15

Czemmel, S., Stracke, R., Weisshaar, B., Cordon, N., Harris, N. N., Walker, A. R., et al. (2009). The grapevine R2R3-MYB transcription factor VvMYBF1
Supplementary Figure S2 | Size distribution of the contigs obtained from de novo assembly of high quality clean reads.

Supplementary Figure S3 | E-value distribution of the BLAST hits for unigenes of $P$. emblica (E-value cut off $1 \mathrm{e}^{-5}$ ).

Supplementary Figure S4 | Conserved domains distribution of the of the best BLAST hits for each unigene.

Supplementary Figure S5 | Similarity distribution of the best BLAST hits for unigenes.

Supplementary Table S1 | Sequences of assembled transcripts of $P$. emblica.

Supplementary Table S2 | Annotation of $\boldsymbol{P}$. emblica transcriptome against NR protein, GO and other databases by BLAST analysis.

Supplementary Table S3 | Details on all the transcription factor (TF) families analyzed in $P$. emblica.

Supplementary Table S4 | Details on all the genes of flavonoid biosynthesis pathway analyzed in $P$. emblica transcriptome.

Supplementary Table S5 | Annotated cytochrome P450s (CYPs) in P. emblica transcriptome.

Supplementary Table S6 | Annotated glycosyltransferases (GTs) in $P$. Emblica transcriptome.

Supplementary Table S7 | Details of all the genes involved in vitamin C biosynthesis identified in $P$. emblica transcriptome.

regulates flavonol synthesis in developing grape berries. Plant Physiol. 151, 1513-1530. doi: 10.1104/pp.109.142059

Dare, A. P., Schaffer, R. J., Lin-Wang, K., Allan, A. C., and Hellens, R. P. (2008). Identification of a cis-regulatory element by transient analysis of co-ordinately regulated genes. Plant Methods 4:17. doi: 10.1186/1746-4811-4-17

Davies, K. M., and Schwinn, K. E. (2003).Transcriptional regulation of secondary metabolism. Funct. Plant Biol. 30, 913-925. doi: 10.1071/FP03062

Diliberto, E. J., and Daniels, A. J. (1991). Multi compartmental secretion of ascorbate and its dual role in dopamine $\beta$-hydroxylation. Am. J. Clin. Nutr. 54, 1163S-1172S

De Tullio, M. C. (2010). The mystery of Vitamin, C. Nat. Educ. 3, 48

Dixon, R. A., and Steele, C. L. (1999). Flavonoids and isoflavonoids - a gold mine for metabolic engineering. Trends Plant Sci. 4, 394-400. doi: 10.1016/S13601385(99)01471-5

Espley, R. V., Hellens, R. P., Putterill, J., Stevenson, D. E., Kutty-Amma, S., and Allan, A. C. (2007). Red colouration in apple fruit is due to the activity of the MYB transcription factor, MdMYB10. Plant J. 49, 414-427. doi: 10.1111/j.1365313X.2006.02964.x

Ferreyra, M. L. F., Rius, S. P., and Casati, P. (2012). Flavonoids: biosynthesis, biological functions, and biotechnological applications. Front. Plant Sci. 3:222 doi: $10.3389 /$ fpls.2012.00222

Fu, L., Niu B., Z., Wu, S., and Li, W. (2012).CD-HIT: accelerated for clustering the next-generation sequencing data. Bioinformatics 28, 3150-3152. doi: 10.1093/bioinformatics/bts565

Grabherr, M. G., Haas, B. J., Yassour, M., Levin, J. Z., Thompson, D. A., Amit, I., et al. (2011). Full-length transcriptome assembly from RNA-seq data without a reference genome. Nat. Biotechnol. 29, 644-652. doi: 10.1038/nbt.1883

Hartmann, U., Sagasser, M., Mehrtens, F., Stracke, R., and Weisshaar, B. (2005). Differential combinatorial interactions of cis-acting elements recognized by R2R3-MYB, BZIP, and BHLH factors control light-responsive and tissuespecific activation of phenylpropanoid biosynthesis genes. Plant Mol. Biol. 57, 155-171. doi: 10.1007/s11103-004-6910-0

Harvey, P. J., Campanella, B. F., Castro, P. M., Harms, H., Lichtfouse, E., Schäffner, A. R., et al. (2002). Phytoremediation of polyaromatic hydrocarbons, anilines and phenols. Environ. Sci. Poll. Res. Int. 9, 29-47. doi: 10.1007/BF02987315

Hernandez, J. M., Heine, G. F., Irani, N. G., Feller, A., Kim, M. G., Matulnik, T. et al. (2004). Different mechanisms participate in the R-dependent activity of 
the R2R3 MYB transcription factor C1. J. Biol. Chem. 279, 48205-48213. doi: 10.1074/jbc.M407845200

Kalra, S., Puniya, B. L., Kulshreshtha, D., Kumar, S., Kaur, J., Ramachandran, S., et al. (2013). De novo transcriptome sequencing reveals important molecular networks and metabolic pathways of the plant, Chlorophytum borivilianum. PLoS ONE 8:e83336. doi: 10.1371/journal.pone.0083336

Kobayashi, S., Ishimaru, M., Hiraoka, K., and Honda, C. (2002). Myb-related genes of the Kyoho grape (Vitis labruscana) regulate anthocyanin biosynthesis. Planta 215, 924-933. doi: 10.1007/s00425-002-0830-5

Krishnaveni, M., and Mirunalini, S. (2010). Therapeutic potential of Phyllanthus emblica (amla): the ayurvedic wonder. J. Basic Clin. Physiol. Pharmacol. 21, 93-105. doi: 10.1515/JBCPP.2010.21.1.93

Kumar, A., Singh, B., Kaur, J., and Singh, K. (2015). Functional characterization of flavanone 3-hydroxylase gene from Phyllanthus emblica (L.). J. Plant Biochem. Biotech. 24, 453-460. doi: 10.1007/s13562-014-0296-0

Kumar, A., and Singh, K. (2012). Isolation of high quality RNA from Phyllanthus emblica and its evaluation by downstream applications. Mol. Biotechnol. 52, 269-275. doi: 10.1007/s12033-011-9492-5

Kumar, G. S., Nayaka, H., Dharmesh, S. M., and Slimath, P. V. (2007). Free and bound phenolic antioxidants in amla (Emblica officinalis) and turmeric (Curcuma longa). J. Food Comp. Anal. 19, 446-452. doi: 10.1016/j.jfca.2005.12.015

Kumar, M. S., Kirubanandan, S., Sripriya, R., and Sehgal, P. K. (2008). Triphala promotes healing of infected full-thickness dermal wound. J. Surg. Res. 144, 94-101. doi: 10.1016/j.jss.2007.02.049

Kumar, S., Kalra, S., Kumar, A., Singh, B., Kaur, J., and Singh, K. (2015). RNASeq mediated root transcriptome analysis of Chlorophytum borivilianum for identification of genes involved in saponin biosynthesis. Funct. Integ. Genomics 16, 37-55. doi: 10.1007/s10142-015-0465-9

Kumaran, A., and Karunakaran, R. J. (2006). Nitric oxide radical scavenging active components from Phyllanthus emblica L. Plant Food Hum. Nutr. 61, 1-5. doi: 10.1007/s11130-006-0001-0

Lin-Wang, K., Bolitho, K., Grafton, K., Kortstee, A., Karunairetnam, S., McGhie, T. K., et al. (2010). An R2R3 MYB transcription factor associated with regulation of the anthocyanin biosynthetic pathway in Rosaceae. BMC Plant Biol. 10:50. doi: 10.1186/1471-2229-10-50

Luan, R. P. (2002). Tyrosine phosphorylation in plant cell signaling. Proc. Natl. Acad. Sci. U.S.A. 99, 11567-11569. doi: 10.1073/pnas.182417599

Lyzenga, W. J., Booth, J. K., and Stone, S. L. (2011). Protein ubiquitination: an emerging theme in Plant abiotic stress tolerance. Am. J. Plant Sci. Biotech. 5, $1-11$.

Mano, J., Belles-Boix, E., Babiychuk, E., Inze, D. , Torii, Y., Hiraoka, E., et al. (2005). Protection against photooxidative injury of Tobacco leaves by 2alkenal reductase detoxication of lipid peroxide-derived reactive carbonyls. Plant Physiol. 139, 1773-1783. doi: 10.1104/pp.105.070391

Mazzucotelli, E., Belloni, S., Marone, D., De Leonardis, A. M., Guerra, D., Di Fonzo, N. L., et al. (2006).The E3 ubiquitin ligase gene family in plants: regulation by degradation. Curr. Genomics 7, 509-522. doi: $10.2174 / 138920206779315728$

Mehrtens, F., Kranz, H., Bednarek, P., and Weisshaar, B. (2005). The Arabidopsis transcription factor MYB12 is a flavonol-specific regulator of phenylpropanoid biosynthesis. Plant Physiol. 138, 1083-1096. doi: 10.1104/pp.104.058032

Miranda-Saavedra, D., and Barton, G. J. (2007). Classification and functional annotation of eukaryotic protein kinases. Proteins 68, 893-914. doi: 10.1002/prot.21444

Misra, B. B. (2014). An updated snapshot of recent advances in transcriptomics and genomics of phytomedicinals. J. Postdoc. Res. 2, 1-14. doi: 10.14304/surya.jpr.v2n2.1

Niu, S. S., Xu, C. J., Zhang, W. S., Zhang, B., Li, X., Lin-Wang, K., et al. (2010). Coordinated regulation of anthocyanin biosynthesis in Chinese bayberry (Myrica rubra) fruit by a R2R3 MYB transcription factor. Planta 231, 887-899. doi: 10.1007/s00425-009-1095-Z

Palapol, Y., Ketsa, S., Lin-Wang, K., Ferguson, I. B., and Allan, A. C. (2009). A MYB transcription factor regulates anthocyanin biosynthesis in mangosteen (Garcinia mangostana L.) fruit during ripening. Planta 229, 1323-1334. doi: 10.1007/s00425-009-0917-3
Perianayagam, J. B., Sharma, S. K., Joseph, A., and Christina, A. J. (2004) Evaluation of anti-pyretic and analgesic activity of Emblica officinalis Gaertn. J. Ethnopharmacol. 95, 83-85. doi: 10.1016/j.jep.2004.06.020

Pertea, G., Huang, X., Liang, F., Antonescu, V., Sultana, R., Karamycheva, S., et al. (2003). TIGR Gene Indices clustering tools (TGICL): a software system for fast clustering of large EST datasets. Bioinformatics 19, 651-652. doi: 10.1093/bioinformatics/btg034

Petrussa, E., Braidot, E., Zancani, M., Peresson, C., Bertolini, A., Patui, S., et al. (2013). Plant flavonoids biosynthesis, transport and involvement in stress responses. Int. J. Mol. Sci. 14, 14950-14973. doi: 10.3390/ijms140714950

Poltanov, E. A., Shikov, A. N., Dorman, H. J., Pozharitskaya, O. N., Makarov, V. G., Tikhonov, V. P., et al. (2009). Chemical and antioxidant evaluation of Indian gooseberry (Emblica officinalis Gaertn, syn. Phyllanthus emblica L). supplements. Phytother. Res. 23, 1309-1315. doi: 10.1002/ptr.2775

Prior, R. L., and Wu, X. (2006). Anthocyanins: structural characteristics that result in unique metabolic patterns and biological activities. Free Radic. Res. 40, 1014-1028. doi: 10.1080/10715760600758522

Rani, A., Singh, K., Ahuja, P. S., and Kumar, S. (2012). Molecular regulation of catechins biosynthesis in tea [Camellia sinensis (L.) O. Kuntze]. Gene 495, 205-210. doi: 10.1016/j.gene.2011.12.029

Ravaglia, D., Espley, R. V., Henry-Kirk, R. A., Andreotti, C., Ziosi, V., Hellens, R. P., et al. (2013). Transcriptional regulation of flavonoid biosynthesis in nectarine (Prunus persica) by a set of R2R3 MYB transcription factors. BMC Plant Biol. 13:68. doi: 10.1186/1471-2229-13-68

Renault, H., Bassard, J. E., Hamberger, B., and Werck-Reichhart, D. (2014). Cytochrome P450-mediated metabolic engineering: current progress and future challenges. Curr. Opin. Plant Biol. 19, 27-34. doi: 10.1016/j.pbi.2014.03.004

Rudrabhatla, P., Reddy, M. M., and Rajasekharan, R. (2006). Genome-wide analysis and experimentation of plant serine/threonine/tyrosine-specific protein kinases. Plant Mol. Biol. 60, 293-319. doi: 10.1007/s11103-005-4109-7

Schuler, M. A. (1996). Plant cytochrome P450 monooxygenases. Crit. Rev. Plant Sci. 15, 235-284. doi: 10.1080/07352689609701942

Schuler, M. A., and Werck-Reichhart, D. (2003). Functional genomics of P450s. Annu. Rev. Plant Biol. 54, 629-667. doi: 10.1146/annurev.arplant.54.031902.134840

Schulz, M. H., Zerbino, D. R., Vingron, M., and Birney, E. (2012). Oases: robust de novo RNA-seq assembly across the dynamic range of expression levels. Bioinformatics 28, 1086-1092. doi: 10.1093/bioinformatics/bts094

Singh, K., Rani, A., Kumar, S., Sood, P., Mahajan, M., Yadav, S. K., et al. (2008). An early gene of the flavonoid pathway, flavanone 3-hydroxylase, exhibits a positive relationship with the concentration of catechins in tea (Camellia sinensis). Tree Physiol. 28, 1349-1356. doi: 10.1093/treephys/28.9.1349

Singh, M. K., Yadav, S. S., Gupta, V., and Khattri, S. (2013). Immunomodulatory role of Emblica officinalis in arsenic induced oxidative damage and apoptosis in thymocytes of mice. BMC Complement. Altern. Med. 13:193. doi: 10.1186/14726882-13-193

Smirnoff, N., and Wheeler, G. L. (2000). Ascorbic acid in plants: biosynthesis and function. Crit. Rev. Biochem. Mol. Biol. 35, 291-314. doi: 10.1080/10409230008984166

Stracke, R., Ishihara, H., Huep, G., Barsch, A., Mehrtens, F., Niehaus, K., et al. (2007). Differential regulation of closely related R2R3-MYB transcription factors controls flavonol accumulation in different parts of the Arabidopsis thaliana seedling. Plant J. 50, 660-677. doi: 10.1111/j.1365-313X.2007.03078.x

Tamaoki, M., Mukai, F., Asai, N., Nakajima, N., Kubo, A., Aono, M., et al. (2003). Light-controlled expression of a gene encoding l-galactono- $\gamma$-lactone dehydrogenase which affects ascorbate pool size in Arabidopsis thaliana. Plant Sci. 164, 1111-1117. doi: 10.1016/S0168-9452(03)00122-5

Tang, Q., Ma, X., Mo, C., Wilson, I. W., Song, C., Zhao, H., et al. (2011). An efficient approach to finding Siraitiagros venorii triterpene biosynthetic genes by RNA-seq and digital gene expression analysis. BMC Genomics 12:343. doi: 10.1186/1471-2164-12-343

Terrier, N., Torregrosa, L., Ageorges, A., Vialet, S., Verries, C., Cheynier, V. et al. (2009). Ectopic expression of VvMybPA2 promotes proanthocyanidin biosynthesis in grapevine and suggests additional targets in the pathway. Plant Physiol. 149, 1028-1041. doi: 10.1104/pp.108.131862 
Werck-Reichhart, D., Hehn, A., and Didierjean, L. (2000). Cytochromes P450 for engineering herbicide tolerance. Trends Plant Sci. 5, 116-123. doi: 10.1016/S1360-1385(00)01567-3

Wheeler, G. L., Mark, A., Jones, M. A., and Smirnoff, N. (1998). The biosynthetic pathway of vitamin C in higher plants. Nature 393, 365-369.

Wilhelm, B. T., Marguerat, S., Watt, S., Schubert, F., Wood, V., Goodhead, I., et al. (2008). Dynamic repertoire of a eukaryotic transcriptome surveyed at single-nucleotide resolution. Nature 453, 1239-1243. doi: 10.1038/nature07002

Winkel-Shirley, B. (2001). Flavonoid biosynthesis. A colorful model for genetics, biochemistry, cell biology, and biotechnology. Plant Physiol. 126, 485-493. doi: 10.1104/pp.126.2.485

Wolucka, B. A., and Van Montagu, M. (2003). GDP-Mannose 30, 50epimerase forms GDP-L-gulose, a putative intermediate for the novo biosynthesis of vitamin C in plants. J. Biol. Chem. 278, 47483-47490. doi: 10.1074/jbc.M309135200
Zerbino, D. R., and Birney, E. (2008). Velvet: algorithms for de novo short read assembly using de Bruijn graphs. Genome Res. 18, 821-829. doi: 10.1101/gr.074492.107

Conflict of Interest Statement: The authors declare that the research was conducted in the absence of any commercial or financial relationships that could be construed as a potential conflict of interest.

Copyright (c) 2016 Kumar, Kumar, Bains, Vaidya, Singh, Kaur, Kaur and Singh. This is an open-access article distributed under the terms of the Creative Commons Attribution License (CC BY). The use, distribution or reproduction in other forums is permitted, provided the original author(s) or licensor are credited and that the original publication in this journal is cited, in accordance with accepted academic practice. No use, distribution or reproduction is permitted which does not comply with these terms. 